\section{Detección, valoración y tratamiento de la hipercolesterolemia en adultos ${ }^{1}$}

Palabras clave: Hipercolesterolemia, síndrome metabólico, factores de riesgo, prevención, farmacoterapia.

\footnotetext{
Basado en el documento "Executive summary of the Third Report of the National Cholesterol Education Program (NCEP) Expert Panel on detection, evaluation and treatment of high blood cholesterol in adults (Adult Treatment Panel III)", disponible en http:// www.nhlbi.nih.gov/guidelines/cholesterol/atp3xsum.pdf
}

Este tercer informe del Grupo de Expertos del Programa Nacional de Educación sobre el Colesterol acerca de la detección, valoración y tratamiento de la hipercolesterolemia en adultos (ATP III) es una actualización de los dos anteriores. En el primero (ATP I) se esbozó la estrategia de prevención primaria de la cardiopatía coronaria (CC) en individuos con concentraciones elevadas $(\geq 160 \mathrm{mg} / \mathrm{dL})$ de colesterol de las lipoproteínas de baja densidad (LDL-C) o concentraciones moderadamente elevadas (130-159 mg/dL) y múltiples $(\geq 2)$ factores de riesgo. En el segundo informe (ATP II) se confirmó la importancia de esta estrategia y se redujo a $\leq 100$ $\mathrm{mg} / \mathrm{dL}$ la concentración de LDL-C deseable en pacientes con CC. Este tercer informe sigue centrándose en el tratamiento de los pacientes con CC, pero propugna un tratamiento más intensivo para reducir el LDL-C en determinados grupos de individuos y presta especial atención a la prevención primaria en pacientes con múltiples factores de riesgo.

\section{EL LDL-C, OBJETIVO PRIMARIO DEL TRATAMIENTO}

Las investigaciones experimentales, epidemiológicas y genéticas han indicado que la elevación del LDL-C es una de las principales causas de CC, y ensayos clínicos recientes han demostrado que los tratamientos que reducen su concentración también reducen el riesgo de CC. Por este motivo, en el ATP III el objetivo primario de los tratamientos hipocolesterolemiantes sigue siendo la reducción del LDL-C.

\section{LA VALORACIÓN DEL RIESGO}

La valoración del riesgo requiere un análisis de las lipoproteínas y la identificación de otros determinantes del riesgo. En adultos de más de 20 años se debería realizar cada 5 años un análisis de las lipoproteínas en ayunas: colesterol total, LDL-C, colesterol de las lipoproteínas de alta densidad (HDL-C) y triglicéridos. Si el análisis no se realiza en ayunas, solo se pueden utilizar los datos del colesterol total y del HDL-C; si el primero es $\geq 200$ $\mathrm{mg} / \mathrm{dL}$ o el segundo es $<40 \mathrm{mg} / \mathrm{dL}$, se debe repetir el análisis en ayunas para disponer de datos sobre el LDL-C. El cuadro 1 muestra la clasificación de los valores de diferentes lipoproteínas. 
CUADRO 1. Clasificación de los valores de diferentes lipoproteínas según el ATP III (en mg/dL)

\begin{tabular}{cccc}
\hline LDL-C & Colesterol total & HDL-C & Triglicéridos \\
\hline $\begin{array}{c}\text { Óptimos } \\
(<100)\end{array}$ & $\begin{array}{c}\text { Deseables } \\
(<200)\end{array}$ & $\begin{array}{c}\text { Bajos } \\
(<40)\end{array}$ & $\begin{array}{c}\text { Normales } \\
(<150)\end{array}$ \\
$\begin{array}{c}\text { Casi óptimos } \\
(100-129)\end{array}$ & & \\
$\begin{array}{c}\text { Moderadamente altos } \\
(130-159)\end{array}$ & $\begin{array}{c}\text { Moderadamente altos } \\
(200-239)\end{array}$ & & $\begin{array}{c}\text { Moderadamente altos } \\
(150-199)\end{array}$ \\
$\begin{array}{c}\text { Altos } \\
(160-189)\end{array}$ & $\begin{array}{c}\text { Altos } \\
(\geq 240)\end{array}$ & $\begin{array}{c}\text { Altos } \\
(\geq 60)\end{array}$ & $\begin{array}{c}\text { Altos } \\
\text { Muy altos } \\
(\geq 190)\end{array}$ \\
\hline
\end{tabular}

Además del LDL-C, los determinantes del riesgo incluyen la presencia o ausencia de CC $\mathrm{u}$ otras formas clínicas de aterosclerosis (arteriopatía periférica, aneurisma aórtico abdominal y arteriopatía carotídea sintomática) y cinco factores de riesgo principales que modifican el tratamiento del LDL-C (cuadro 2). Se distinguen tres categorías de riesgo que modifican las concentraciones deseables de LDL-C (cuadro 3).

\section{Método de valoración del riesgo: recuento de los principales factores de riesgo y estimación del riesgo de CC a los 10 años}

El riesgo de las personas sin CC $\mathrm{u}$ otras formas de enfermedad aterosclerótica clínicamente

CUADRO 2. Factores de riesgo principales, que modifican el objetivo terapéutico del colesterol de las lipoproteínas de baja densidad ${ }^{a}$

Consumo de tabaco

Hipertensión (tensión arterial $\geq 140 / 90$ mm Hg o medicación antihipertensiva)

HDL-C ${ }^{b}$ bajo $(<40 \mathrm{mg} / \mathrm{dL})^{\mathrm{c}}$

Antecedentes familiares de cardiopatía coronaria prematura (cardiopatía coronaria en un familiar en primer grado antes de los 55 años en hombres 0 antes de los 65 en mujeres)

Edad ( $\geq 45$ años en el hombre $y \geq 55$ en la mujer)

a La diabetes no se incluye en este grupo, pues se considera como un equivalente de la CC por lo que al riesgo se refiere (equivalente de riesgo).

b HDL-C: colesterol de las lipoproteínas de alta densidad.

c El HDL-C alto ( $\geq 60 \mathrm{mg} / \mathrm{dL}$ ) se considera un factor de riesgo "negativo" (protector) y su presencia elimina del recuento a uno de los demás. manifiestas se determina por un procedimiento que consta de dos pasos: 1) el recuento del número de factores de riesgo, y 2) en personas con múltiples $(\geq 2)$ factores de riesgo, la valoración del riesgo a los 10 años con el sistema de puntuación de Framingham, en el que se incluyen la edad, el colesterol total, el HDL-C, la tensión arterial y el consumo de tabaco, y que divide a estos individuos en tres categorías con respecto al riesgo de CC a los 10 años: $>20 \%, 10-20 \%$ y $<10 \%$.

\section{Papel de otros factores en la valoración del riesgo}

Además de los cinco factores de riesgo principales antes mencionados, existen otros factores que influyen en el riesgo de CC. Entre ellos se encuentran los factores de riesgo relacionados con los hábitos de vida (obesidad, inactividad física y dieta aterógena) y los llamados factores de riesgo emergentes (lipoproteína (a), homocisteína, factores protrombóticos y proinflamatorios, elevación de la glucemia en ayunas y signos de enfermedad aterosclerótica subclínica).

\section{Síndrome metabólico}

Muchas personas presentan diferentes combinaciones de factores de riesgo principales, factores de riesgo relacionados con los hábitos de vida y factores de riesgo emergentes, que constituyen el llamado síndrome metabólico. Los factores más característicos de este síndrome son la obesidad abdominal, la dislipidemia aterógena, la hiperten- 
CUADRO 3. Categorías de riesgo que modifican las concentraciones deseables de colesterol de las lipoproteínas de baja densidad (LDL-C) y riesgo de accidente coronario importante a los 10 años

\begin{tabular}{lcc}
\hline \multicolumn{1}{c}{ Categoría de riesgo } & $\begin{array}{c}\text { LDL-C deseable } \\
(\mathrm{mg} / \mathrm{dL})\end{array}$ & $\begin{array}{c}\text { Riesgo a los 10 años } \\
(\%)\end{array}$ \\
\hline CC $^{a}$ y equivalentes de riesgo & $<100$ & $>20$ \\
Múltiples $(\geq 2)$ factores de riesgo & $<130$ & $\leq 20$ \\
$0-1$ factor de riesgo & $<160$ & $<10$ \\
\hline
\end{tabular}

a CC: cardiopatía coronaria.

sión, la resistencia a la insulina (con o sin intolerancia a la glucosa) y los estados protrombóticos y proinflamatorios. El ATP III reconoce este síndrome como un objetivo secundario del tratamiento de reducción del riesgo. El diagnóstico del síndrome metabólico se establece en presencia de tres o más de los determinantes de riesgo enumerados en el cuadro 4.

\section{PREVENCIÓN PRIMARIA CON TRATAMIENTOS PARA REDUCIR EL LDL-C}

La prevención primaria de la CC, cuyo objetivo consiste en reducir el riesgo tanto a corto $(\leq 10$ años) como a largo plazo (> 10 años), está basada principalmente en cambios del estilo de vida consistentes en la reducción de la ingesta de grasas saturadas y colesterol, el aumento de la actividad física y el control del peso. No obstante, en los individuos con mayor riesgo debido a la presencia de concentraciones elevadas o muy elevadas de LDL-C o de múltiples factores de riesgo también puede ser necesario un tratamiento farmacológico para reducir el LDL-C, cuyos valores deseables dependen del riesgo absoluto de CC. Antes de iniciar un

\section{CUADRO 4. Criterios para el diagnóstico del síndrome me- tabólico ${ }^{\mathrm{a}}$}

Obesidad abdominal (perímetro de la cintura $>102 \mathrm{~cm}$ en el hombre $y>88 \mathrm{~cm}$ en la mujer)

Triglicéridos $\geq 150 \mathrm{mg} / \mathrm{dL}$

HDL-C ${ }^{b}<40 \mathrm{mg} / \mathrm{dL}$ en el hombre $0<50 \mathrm{mg} / \mathrm{dL}$ en la mujer

Tensión arterial $\geq 130 / \geq 85 \mathrm{~mm} \mathrm{Hg}$

Glucemia en ayunas $\geq 110 \mathrm{mg} / \mathrm{dL}$

\footnotetext{
a El diagnóstico se establece en presencia de tres o más de estos determinantes de riesgo.

b HDL-C: colesterol de las lipoproteínas de alta densidad.
}

tratamiento destinado a reducir los lípidos, toda persona con LDL-C elevado o alguna otra forma de hiperlipidemia debe ser sometida a estudios clínicos y de laboratorio para descartar la presencia de causas de dislipidemia secundaria, como la diabetes, el hipotiroidismo, la hepatopatía obstructiva, la insuficiencia renal crónica o los fármacos que aumentan el LDL-C y reducen el HDL-C (progestágenos, esteroides anabolizantes y corticoesteroides). Una vez que se han excluido o tratado adecuadamente estas causas secundarias, se establecerán los objetivos del tratamiento de reducción del LDL-C en función de la categoría de riesgo del individuo.

\section{PREVENCIÓN SECUNDARIA CON TRATAMIENTOS PARA REDUCIR EL LDL-C}

Ensayos clínicos recientes han demostrado que el tratamiento para reducir el LDL-C reduce la mortalidad total, la mortalidad por causas coronarias, los accidentes coronarios importantes, los procedimientos coronarios y los accidentes vasculares cerebrales en individuos con CC establecida. El objetivo de la prevención secundaria en lo que se refiere a la concentración de LDL-C es de $<100 \mathrm{mg} / \mathrm{dL}$, cifra respaldada por los ensayos clínicos con medidas de eficacia tanto clínicas como angiográficas y por los estudios epidemiológicos prospectivos. Ese mismo objetivo se aplica también a los equivalentes de riesgo.

\section{TRATAMIENTOS PARA REDUCIR EL LDL-C EN LAS TRES CATEGORÍAS DE RIESGO}

Las dos modalidades terapéuticas principales para reducir el LDL-C son los cambios terapéuticos del estilo de vida y la farmacoterapia. Las concentraciones de LDL-C en las que hay que considerar la necesidad de tratamiento farmacológico dependen de la categoría de riesgo (cuadro 5). 
CUADRO 5. Concentraciones de colesterol de las lipoproteínas de baja densidad (LDL-C) que requieren considerar la necesidad de tratamiento farmacológico en función de la categoría de riesgo

\begin{tabular}{ll}
\hline \multicolumn{1}{c}{ Categoría de riesgo } & \multicolumn{1}{c}{ LDL-C (mg/dL) } \\
\hline CC $^{a}$ o equivalentes de riesgo & $\geq 130$ \\
& $($ entre 100 y 129 es optativo) \\
Múltiples $(\geq 2)$ factores de riesgo & $\geq 130$ si el riesgo a los 10 años es del $10-20 \%$ \\
& $\geq 160$ si el riesgo a los 10 años es $<10 \%$ \\
$0-1$ factor de riesgo & $\geq 190$ \\
& (entre 160 y 189 es optativo) \\
\hline
\end{tabular}

a CC: cardiopatía coronaria.

\section{CAMBIOS TERAPÉUTICOS DEL ESTILO DE VIDA EN EL TRATAMIENTO PARA REDUCIR EL LDL-C}

El primero de estos cambios que se debe instaurar para reducir el LDL-C es la reducción de la ingesta de grasas saturadas $(<7 \%$ de las calorías totales) y colesterol (<200 mg/día). Si al cabo de 6 semanas no se ha alcanzado la concentración deseada de LDL-C, se pueden añadir otras opciones terapéuticas que potencian la reducción de las LDL, como los estanoles y esteroles vegetales ( $2 \mathrm{~g} /$ día $)$ o el aumento de las fibras viscosas (10-25 g/día). Tras la obtención de la máxima reducción posible con las medidas dietéticas, el énfasis cambia hacia el tratamiento del síndrome metabólico y de los factores de riesgo lipídicos asociados, con medidas como la reducción de peso y el aumento de la actividad física. La composición de la dieta recomendada con los cambios terapéuticos del estilo de vida se muestra en el cuadro 6.

\section{TRATAMIENTO FARMACOLÓGICO PARA ALCANZAR LA CONCENTRACIÓN DESEADA DE LDL-C}

En los casos que también necesiten tratamiento farmacológico no se deben olvidar las medidas relacionadas con los cambios terapéuticos del estilo de vida. En la actualidad existen cuatro clases de fármacos que modifican el metabolismo de las lipoproteínas: las estatinas (lovastatina, pravastatina, simvastatina, fluvastatina, atorvastatina y cerivastatina), los secuestradores de ácidos biliares (colestiramina, colestipol y colesevelam), el ácido nicotínico y los derivados del ácido fíbrico (gemfibrozilo, fenofibrato y clofibrato).

CUADRO 6. Composición de la dieta recomendada con los cambios terapéuticos del estilo de vida

\begin{tabular}{ll}
\hline \multicolumn{1}{c}{ Componente } & \multicolumn{1}{c}{ Cantidad } \\
\hline Grasas saturadas & $<7 \%$ de las calorías totales \\
Grasas poliinsaturadas & Hasta un $10 \%$ de las calorías totales \\
Grasas monoinsaturadas & Hasta un $20 \%$ de las calorías totales \\
Grasas totales & Hasta un $25-35 \%$ de las calorías totales \\
Hidratos de carbono & $50-60 \%$ de las calorías totales \\
Fibra & $20-30$ g/día \\
Proteínas & Aproximadamente $15 \%$ de las calorías totales \\
Colesterol & $<200$ mg/día \\
Calorías totales & Equilibrio entre el ingreso y el gasto, para mantener el \\
& peso corporal deseable y evitar su aumento \\
\hline
\end{tabular}




\section{Prevención secundaria: farmacoterapia para la CC y equivalentes de riesgo}

La mayoría de los pacientes con CC o equivalentes de riesgo necesitan tratamiento farmacológico. Otros factores de riesgo lipídicos también pueden justificar la consideración de este tratamiento. Se usen o no fármacos modificadores de los lípidos, sigue siendo necesario prestar atención a los factores de riesgo no lipídicos y modificarlos. Al darlos de alta, los pacientes hospitalizados por accidente coronario importante o para la realización de un procedimiento coronario deben abandonar el hospital con un tratamiento farmacológico si su concentración de LDL-C es $\geq 130 \mathrm{mg} / \mathrm{dL}$; si es de 100-129 mg/dL, el tratamiento es optativo, pero hay que tener en cuenta que el LDL-C empieza a disminuir en las primeras horas después de un accidente coronario, que a las $24-48$ horas se encuenta significativamente disminuido y que puede mantenerse bajo durante muchas semanas. Por consiguiente, la concentración inicial de LDL-C registrada en el hospital puede ser muy inferior a la habitual en el paciente.

\section{Farmacoterapia para reducir el LDL-C en la prevención primaria}

Cuando se considere la necesidad de farmacoterapia en la prevención primaria, el tratamiento debe comenzar generalmente durante la tercera consulta tras el inicio del tratamiento dietético. Aunque se inicie un tratamiento farmacológico, no se deben abandonar los cambios terapéuticos del estilo de vida. Los fármacos más utilizados son las estatinas, pero también se pueden utilizar secuestradores de los ácidos biliares o ácido nicotínico. En la mayoría de los casos se debe comenzar con dosis moderadas de estatinas. La respuesta al tratamiento debe ser valorada a las 6 semanas y si se han alcanzado los objetivos terapéuticos se mantiene la dosis; en caso contrario, se intensificará el tratamiento, bien aumentando la dosis de estatina o combinándola con un secuestrador de los ácidos biliares o con el ácido nicotínico. La respuesta al tratamiento debe ser valorada nuevamente 6 semanas más tarde y, a partir de entonces, cada 4 a 6 meses, o más frecuentemente si se considera necesario.

\section{EL SÍNDROME METABÓLICO COMO OBJETIVO TERAPÉUTICO SECUNDARIO}

Este síndrome está estrechamente relacionado con un trastorno metabólico generalizado, denomi- nado resistencia a la insulina. El exceso de grasa corporal (en particular la obesidad abdominal) y la inactividad física promueven la aparición de la resistencia a la insulina, pero en algunos individuos también existe una predisposición genética. El tratamiento del síndrome metabólico tiene un doble objetivo: atenuar las causas subyacentes (obesidad e inactividad física) y tratar los factores de riesgo lipídicos y no lipídicos asociados.

\section{Tratamiento de las causas subyacentes del síndrome metabólico}

Los tratamientos de primera línea para todos los factores de riesgo lipídicos y no lipídicos asociados al síndrome metabólico son la reducción del peso y el aumento de la actividad física.

\section{Tratamiento específico de los factores de riesgo lipídicos y no lipídicos}

En este apartado se incluyen el tratamiento de la hipertensión, la administración de aspirina en pacientes con CC a fin de reducir el estado protrombótico y el tratamiento de la hipertrigliceridemia y de las bajas concentraciones de HDL-C.

\section{PROBLEMAS ESPECIALES}

\section{Tratamiento de dislipidemias específicas}

LDL-C muy elevado ( $\geq 190 \mathrm{mg} / \mathrm{dL}$ ). Las personas con concentraciones muy elevadas de LDL-C tienen generalmente formas genéticas de hipercolesterolemia, como hipercolesterolemia familiar monogénica, apoliproteína B defectuosa familiar o hipercolesterolemia poligénica. Para evitar la CC prematura es necesaria la detección temprana de estos trastornos mediante determinaciones del colesterol. También es importante realizarles estas pruebas a los familiares para identificar otros afectados. Estos trastornos suelen necesitar farmacoterapia combinada (una estatina más un secuestrador de los ácidos biliares).

Hipertrigliceridemia. Los factores que contribuyen a la existencia de concentraciones de triglicéridos superiores a las normales incluyen la obesidad y el sobrepeso, la inactividad física, el consumo de tabaco, el consumo excesivo de alcohol, las dietas ricas en hidratos de carbono ( $>60 \%$ de las calorías totales) y varias enfermedades (diabetes de tipo II, insuficiencia renal crónica, síndrome nefrótico), fár- 
macos (corticoesteroides, retinoides, altas dosis de $\beta$-bloqueantes) y trastornos genéticos (hiperlipidemia combinada familiar, hipertrigliceridemia familiar y disbetalipoproteinemia familiar).

En personas con triglicéridos elevados $(>200$ $\mathrm{mg} / \mathrm{dL}$ ), el ATP III considera como un objetivo secundario del tratamiento a la suma del LDL-C más el colesterol de las lipoproteínas de muy baja densidad (VLDL-C), también llamado colesterol no de las HDL (colesterol total - HDL-C), cuya cifra debe ser, como máximo, $30 \mathrm{mg} / \mathrm{dL}$ mayor que la del LDL-C, partiendo del principio de que el VLDL-C normal es $\leq 30 \mathrm{mg} / \mathrm{dL}$.

La estrategia terapéutica recomendable en caso de elevación de los triglicéridos depende de su causa y de su gravedad. En toda persona con hipertrigliceridemia, el objetivo primario del tratamiento es alcanzar la concentración deseada de LDL-C. Cuando los triglicéridos están moderadamente elevados (150-199 mg/dL), se debería prestar especial atención a la pérdida de peso y al aumento de la actividad física. Cuando están elevados (200-499 mg/dL), el colesterol no de las HDL constituye un objetivo secundario del tratamiento y, además de la pérdida de peso y el aumento de la actividad física, se puede considerar la necesidad de farmacoterapia para alcanzar la concentración deseada, lo cual se puede hacer intensificando el tratamiento con un fármaco que reduzca el LDL-C, o añadiendo ácido nicotínico o fibrato. En los raros casos en los que los triglicéridos están muy elevados ( $\geq 500 \mathrm{mg} / \mathrm{dL}$ ) el objetivo prioritario del tratamiento es reducir los triglicéridos para evitar la pancreatitis aguda, para lo cual se necesita una dieta en la que las grasas representen $\leq 15 \%$ de la ingesta calórica, reducir el peso, aumentar la actividad física $\mathrm{y}$, generalmente, un fármaco que reduzca los triglicéridos (fibrato o ácido nicotínico). Después de haber logrado concentraciones de triglicéridos $<500 \mathrm{mg} / \mathrm{dL}$, la atención debería centrarse en la reducción del LDL-C, para disminuir el riesgo de CC.

HDL-C bajo. El HDL-C bajo ( $<40 \mathrm{mg} / \mathrm{dL})$ está estrechamente relacionado con la CC. Sus causas son diversas y muchas de ellas se asocian a la resistencia a la insulina: triglicéridos elevados, sobrepeso y obesidad, inactividad física y diabetes de tipo II. Otras causas son el consumo de tabaco, las ingestas muy ricas en carbohidratos ( $>60 \%$ de las calorías totales) y algunos fármacos, como los $\beta$-bloqueantes, esteroides anabolizantes y progestágenos. El ATP III no especifica un objetivo terapéutico en la elevación del HDL-C porque no existen datos suficientes para hacerlo y porque los fármacos actuales no son muy eficaces para aumentar el HDL-C.
Dislipidemia diabética. Dado que el ATP III considera la diabetes como un equivalente de riesgo de la CC, el objetivo terapéutico primario en la mayoría de los pacientes consiste en reducir la concentración de LDL-C a $<100 \mathrm{mg} / \mathrm{dL}$. Cuando el LDL-C es $>130 \mathrm{mg} / \mathrm{dL}$, para conseguir este objetivo la mayoría de las personas con diabetes necesitan farmacoterapia añadida a los cambios terapéuticos del estilo de vida. Cuando se encuentra en el intervalo de $100-129 \mathrm{mg} / \mathrm{dL}$ en condiciones basales o durante el tratamiento, existen varias opciones terapéuticas: intensificar el tratamiento de reducción del LDL-C, añadir un fármaco para modificar la dislipidemia aterógena (fibrato o ácido nicotínico) o intensificar el control de otros factores de riesgo, incluida la diabetes. Cuando los triglicéridos son $\geq 200 \mathrm{mg} / \mathrm{dL}$, el colesterol no de las HDL representa un objetivo terapéutico secundario. En personas de $\geq 65$ años con diabetes, pero sin otros factores de riesgo de CC que no sean la edad, la aplicación de estas normas dependerá del juicio clínico, pues existen muchos otros factores (enfermedades concomitantes, estado de salud general, aspectos sociales) que pueden influir en las decisiones terapéuticas e indicar un tratamiento más conservador.

\section{Consideraciones especiales en diferentes grupos de población}

Hombres de mediana edad (35-65 años). En general, los hombres corren mayor riesgo de sufrir CC que las mujeres. En particular, los hombres de mediana edad tienen una alta prevalencia de los principales factores de riesgo y están predispuestos a la obesidad abdominal y al síndrome metabólico. Por consiguiente, corren un riesgo relativamente alto de sufrir $\mathrm{CC}$ y, cuando esto realmente ocurre, necesitan tratamientos intensivos para reducir el LDL-C.

Mujeres (45-75 años). En las mujeres, la aparición de CC ocurre generalmente 10-15 años después que en los hombres. Aunque esta diferencia se ha atribuido al efecto protector de los estrógenos, estudios recientes de prevención primaria y secundaria han puesto en duda la eficacia del tratamiento de reposición de estrógenos para reducir el riesgo de CC en mujeres posmenopáusicas. En cambio, los ensayos clínicos han puesto de manifiesto los efectos del tratamiento con estatinas. En la prevención secundaria, las mujeres deben ser tratadas de la misma forma que los hombres. En la prevención primaria, el abordaje general del ATP III también es aplicable tanto a los hombres como a las mujeres, pero las decisiones clínicas sobre el tratamiento farmacológico 
deben tener en cuenta el inicio generalmente más tardío de la CC en las mujeres.

Adultos de edad avanzada (hombres de más de 65 años y mujeres de más de 75 años). En estos individuos, las concentraciones elevadas de LDL-C y las concentraciones bajas de HDL-C siguen teniendo valor predictivo con respecto a la aparición de CC. No obstante, la identificación de signos de aterosclerosis subclínica avanzada mediante pruebas incruentas también puede ayudar a confirmar la presencia de un riesgo elevado. Los ensayos clínicos de prevención secundaria con estatinas han demostrado una significativa reducción del riesgo. En la prevención primaria, las medidas iniciales consisten en los cambios terapéuticos del estilo de vida, aunque esto no significa que no se deba considerar la necesidad de tratamiento farmacológico en individuos con alto riesgo debido a la presencia de múltiples factores de riesgo o de aterosclerosis subclínica avanzada.

Adultos jóvenes (hombres de 20-35 años y mujeres de 20-45 años). Aunque la CC es rara en estos individuos, excepto en aquellos con factores de riesgo graves (hipercolesterolemia familiar, gran consumo de tabaco o diabetes), la identificación de los factores de riesgo en adultos jóvenes es muy importante para la prevención a largo plazo. Cuando el LDL-C es $\geq 130 \mathrm{mg} / \mathrm{dL}$ se deben introducir cambios terapéuticos del estilo de vida. Los hombres jóvenes, fumadores, con LDL-C elevado (160-189 $\mathrm{mg} / \mathrm{dL}$ ) requieren especial atención y pueden ser candidatos al tratamiento farmacológico. Cuando el LDL-C es muy elevado ( $\geq 190 \mathrm{mg} / \mathrm{dL}$ ), se debe considerar el tratamiento farmacológico, como en cualquier otro adulto. La presencia de formas genéticas graves de hipercolesterolemia puede necesitar tratamiento farmacológico combinado (por ejemplo, una estatina más un secuestrador de los ácidos biliares).

Grupos raciales y étnicos. Los afroamericanos son el grupo étnico con mayor tasa de mortalidad global por CC en los Estados Unidos de América, sobre todo en jóvenes. Aunque sus causas no están totalmente esclarecidas, este fenómeno se puede explicar, al menos parcialmente, por la alta prevalencia de factores de riesgo coronarios. La hipertensión, la hipertrofia ventricular izquierda, la diabetes, el tabaquismo, la obesidad, la inactividad física y la combinación de múltiples factores de riesgo de CC son más frecuentes en los afroamericanos que en los blancos. Aunque existen datos que señalan diferencias en el riesgo basal de $\mathrm{CC}$ de otros grupos raciales y étnicos, son insuficientes para modificar las recomendaciones generales.

\section{Adherencia al tratamiento para reducir el LDL-C}

La adherencia a las recomendaciones del ATP III por parte de los pacientes y de los profesionales sanitarios es la clave para poder acercarse en la práctica clínica a los beneficios obtenidos en los ensayos clínicos sobre la reducción del colesterol. Entre las numerosas intervenciones que pueden mejorar el cumplimiento del tratamiento se encuentran la simplificación de los regímenes terapéuticos, la educación del paciente sobre cómo seguir el tratamiento, el apoyo de los familiares y amigos, el refuerzo y la recompensa por el cumplimiento, la educación del médico para que ponga en práctica las directrices terapéuticas, la creación de planes terapéuticos uniformizados, la creación de consultas especializadas en los trastornos de los lípidos o el desarrollo de la telemedicina.

\section{SYNOPSIS}

\section{Detection, evaluation, and treatment of high blood cholesterol in adults}

This report is based on the Third Report of the National Cholesterol Education Program (NCEP) Expert Panel on Detection, Evaluation, and Treatment of High Blood Cholesterol in Adults, which was recently issued by the National Institutes of Health of the United States of America. Also known as the Adult Treatment Panel (ATP) III, this new report updates two earlier such reports on high cholesterol. While continuing to concentrate on treating patients with coronary heart disease, the new report advocates more intensive treatment in order to reduce low-density lipoprotein (LDL) cholesterol in specific groups of individuals, pays special attention to primary prevention among patients with multiple risk factors, and recognizes as a secondary prevention concern a cluster of heart disease risk factors known as "the metabolic syndrome." Other issues that the ATP III report covers include therapeutic lifestyle changes to reduce $L D L, L D L$-lowering drug therapy, and the management of specific dyslipidemias. 